\title{
UNIVERSIDAD, VINCULACIÓN CON LA SOCIEDAD Y PROCESOS DE MEJORAMIENTO
}

\author{
UNIVERSITY, LINK TO SOCIETY AND IMPROVEMENT PROCESS \\ Gonzalo Junior Chávez Cruz* \\ Unidad de Posgrado de la Facultad de Ciencias Contables \\ Universidad Nacional Mayor de San Marcos - UNMSM / Lima - Perú \\ [Recepción: Abril de 2016/ Conformidad: Mayo 2016]
}

\section{RESUMEN}

Este artículo analiza la Ley Orgánica de Educación Superior del Ecuador en lo que respecta a la vinculacióny los aspectos que obligan a las instituciones de educación superior a actuar ante las expectativas y necesidades de la sociedad, segúnla planificación nacional, el régimen de desarrollo, el perfeccionamiento científico, humanístico y tecnológico mundial, y la diversidad cultural presente en el país. En función de esto, se describe la importancia de la vinculación con la sociedad y se resalta esta acción como el indicador más fuerte de categorización de las universidades en los actuales momentos. Para ello, se brindan algunas ideas de cómo debería estructurarse un modelointegral de trabajo paralograr una efectiva vinculación entre la universidad, empresas y la sociedad, utilizando como referente la Unidad Académica de Ciencias Empresariales.

\section{Palabras Clave:}

Vinculación; empresas; educación superior; estrategias metodológicas; sociedad del conocimiento.

\begin{abstract}
This article analyzes the Organic Law on Higher Education of Ecuador with regards to the links and the aspects that force the Higher Education Institutions to act on the expectations and needs of the community, considering the national planning, the development regime, the worldwide scientific, humanistic and technological improvement, and the cultural diversity in the country.

The importance of the relation with the community is described and it is highlighted as the strongest indicator of universities categorization at the present time. For that effect, some ideas are provided on how a comprehensive working model should be structured for effective linkage among the university, business and community, using as reference the Academic Unit of Business Science.
\end{abstract}

\section{Keywords:}

Linkage; businesses; higher education; methodology strategies; knowledge society.

\footnotetext{
* Doctorando de Ciencias Contables y Empresariales - UNMSM. Email: gonzach123@hotmail.com
} 


\section{INTRODUCCIÓN}

Los debates sobre la calidad de la educación superior son cada vez más frecuentes en Ecuador. Estos coinciden en afirmar que el mejoramiento de la calidad constituye un campo de difícil definición e intervención debido a la gravedad con que está afectada a nivel institucional. Desde la promulgación de la Ley Orgánica de Educación Superior, (Asamblea Nacional del Ecuador, 2010), los centros de enseñanza superior se han visto abocados a una serie de cambios estructurales en sus comportamientos habituales y han pasado de ser instituciones que mantenían objetivos propiosa responder a un marco institucional coherente con el Estado, según actividades que antes no eran apreciadas por las universidades. Esto es evidente en el caso de la Universidad Técnica de Machala, específicamente, en la Unidad Académica de Ciencias Empresariales. En ella, las acciones que se venían realizando en cuanto a acercamientos con empresas y organizaciones no reunían los requisitos necesarios para poder medir los resultados de esta gestión. Se actuaba en función de procesos coyunturales, dejando de lado la acción estructural, que conlleva planificar procesos de inserción universitaria. tanto en la colectividad como en las organizaciones empresariales públicas y privadas. En este contexto, se determinaba un rumbo entre lo que buscado por los actores externos frente a lo ofrecido por la universidad y, en especial, la Unidad Académicas de Ciencias Empresariales. De ese modo, se planificaban acciones solo por cumplir con ellas antes que para estructurar un acercamiento técnico yprofesional con la comunidad. Evidencia de ello, es la firma de convenios de vinculación sin el correspondiente diseño de mecanismos de seguimiento y evaluación de estos convenios.

Hoy día la vinculación de la Unidad Académica de Ciencias Empresariales (UACE) de la Universidad Técnica de Machala (UTMACH) con el sector empresarial, público y privado, es un asunto de vital importancia, puesto que tiene como objetivo vincular las seis carreras que ella oferta con el sector empresarial de la provincia de El Oro. Antes de la publicación de la nueva Ley Orgánica de Educación Superior (LOES) (Asamblea Nacional del Ecuador, 2010), se trató de realizar esta tarea mediante planes de extensión universitaria. Así, ante la falta de conexión con los problemas de la sociedad se hicieron convenios, pasantías, seminarios y conferencias para estrechar o fundar este nexo. Más adelante,para la aplicación de este mandato de ley en la UACE y para crear un nexo con la colectividad, se crearon dos departamentos (uno de vinculación y otro de pasantías) y se nombró un responsable para dirigir este proceso (sin nombramiento y sin horas asignadas).

A raíz de lo mencionado, la existencia de una política de vinculación en esta oficina académica puede entenderse como resultado de un acto de cumplimiento legal que dista de ser claramente pensada y estructurada desde mucho tiempo atrás, es decir, un esfuerzo que, como se mencionó,adolece de herramientas para poder evaluar sus impactos y retroalimentar los resultados. Esta es una seria limitación que impide un buen desempeño de acuerdo al indicador de vinculación con la colectividad planteado por el gobierno (Consejo de Evaluación, Acreditación y Aseguramiento de la Calidad de la Educación CEAACES, 2015), una situación que puede ser tomada como una realidad común en muchas universidades públicas de Ecuador.

\section{MARCO PARA EL ANÁLISIS}

El punto de partida de este análisis es la Ley Orgánica de Educación Superior del Ecuador(LOES. Art. 13. Literal a., 2010). En ella se establecen las funciones del sistema de educación superior, entre las cuales se menciona que sedebe garantizar el derecho a la educación superior mediante la docencia, la investigación y su vinculación con la sociedad, y asegurar,de ese modo, crecientes niveles de calidad y excelencia académica entre los profesionales del país. De acuerdo con esto, se entiende que las Instituciones de Educación Superior (IES) deben en todo momento vincularse mediante una acción dialógica con la comunidad, sin desconocer el rol de la empresa privada y pública (miembro de la tríada de desarrollo de un territorio). Así, pues, se propugna la implementación de procesos intra y extrauniversitarios mediados por la praxis y no por la teoría, a partir de los cuales prima la atención a las necesidades de las personas en sus territorios antes que las discusiones al interior de la academia. 
Según lo observado, es la misma LOES la que determina que la acreditación de fondos para las universidades debe considerar la vinculación de su oferta académica al desarrollo nacional o regional y su aporte a la creación de sinergias, asociaciones o fusiones con otras instituciones de educación superior de la región, sin olvidar la promoción de laspotencialidades territoriales. Esto es lo que obliga a las universidades a mirar fuera del cerco académico en busca de alianzas estratégicas que les permitan crecer, fomentar y generar el bien común uno de los lineamientos básicos en el Plan Nacional del Buen Vivir(PNBV, 2013), y desarrollar nuevos conocimientos interdisciplinarios en consideración de los saberes ancestrales presentes en los territorios que son objeto de su intervención.

Ahora bien, ¿qué ha llevado a que se propongan tan radicales cambios en la academia? Vale revisar las influencias de legislaciones y convenciones internacionales, reformas académicas y los acelerados cambios sociales, para advertir que de ese modo se está señalando el rumbo de la academia hacia metas solidarias antes que privadas y ausentes de interés público.

\section{VISIÓN HISTÓRICA DE LOS CAMBIOS EDUCATIVOS}

\section{Primera Reforma (1920 - 1960)}

El periodo temporal a analizar plantea una serie de cambios en la sociedad latinoamericana, algunos de ellos marcados por la crisis económica y otros por los cambios tecnológicos ocurridos. Así, se puede señalar al fenómeno migratorio del campo a la ciudad como uno de los más importantes. A ello se suma la presencia de modelos de industrialización que nacen después de la Segunda Guerra Mundial, que conducen a que haya una necesidad de mano de obra calificada que atienda a ese proceso industrializador, lo que a su vez presupone una universidad orientada a las necesidades empresariales.

En este periodo pocos fueron los trabajos o actividades hechos en torno a la atención de las actividades sociales, rurales o comunitarias, por lo que se puede colegir que se trabajó al margen de las necesidades concretas de los sectores demandantes de ayuda por parte del Estado. Ya en 1918 la Universidad de Córdova en Argentina asumió la responsabilidad de generar el gran cambio en la concepción de la universidad que se requería. Este movimiento trasformador de las estructuras políticas universitarias se encaminó a fortalecer la función social de esta y proyectar la cultura universitaria al pueblo y la solución de los problemas nacionales, mediante la extensión universitaria. Para Peña (2010), quien cita a su vez a Torres \&Trápaga (2010), las universidades deben constituirse no solo como centros formadores de ciudadanos, sino como instituciones generadoras de ideas y propuestas para mejorar las funciones y estructuras sociales.

La reforma de Córdova confirmó la concepción de la educación como un derecho humano y como un deber público que debían promover los estados a través de las universidades. Esto es lo que en los tiempos actuales el organismo rector ecuatoriano denominado Consejo de Educación Superior (CES), junto con su ejecutor la Secretaría Nacional Educación Superior de Ciencia y Tecnología (SENESCYT), viene trabajando, por un lado,tanto a nivel del acceso gratuito a la educación superior como dela depuración de universidades sin estándares de calidad, y, por el otro, a través de una legislación que busca acreditar IES bajo parámetros exigentes, uno de los cuales es justamente el de vinculación.

Desde la reforma de Córdova a la presente fecha, más de 90 años se han necesitado en el Ecuador para dar intentar dar el salto a la calidad y a la definición de educación como un deber público, sin olvidar la autonomía universitaria. De ese modo, se busca volverlas nuevamente protagonistas, ya no solo de las luchas sociales, sino de las transformaciones científicas, conservando el estado de satisfacción de sus clientes internos y externos, si se puede tomar este concepto empresarial de planificación. Para eso, se apela a los saberes ancestrales, al respeto del medio ambiente, la revaloración de las costumbres y, sobre todo, a la responsabilidad social de la universidad frente a la sociedad.

Se aprecia, entonces, que con ciertas experiencias la primera reforma no logró adentrarse en el pensamiento universitario en su universo. Los avances se produjeron lentamente y es en la década de los 60 
cuando surgió de manera oficial en Latinoamérica la tercera función de la universidad: la extensión, vinculada a la educación popular. Esto implicó entender ala vinculación como la forma de abrirse a las necesidades territoriales, un objetivo para el cual en Ecuador se crearon institutos agrícolas que buscaron brindar servicios de capacitación, investigación y desarrollo en zonas agrícolas rurales.

\section{Segunda Reforma (1970 - 1990)}

En este periodo se puede mencionar el fracaso de la propuesta universitaria orientada al crecimiento económico. Ello se debió a que los intentos de vinculación resultaron aislados y fueron reducidos en virtud de los lineamientos de un modelo neoliberal empresarial que debía sustentarse en una universidad que cede espacio a la presencia de competidores privados. Para ello, se comenzó a minar la representatividad del accionar de la universidad pública y a apologizar los trabajos privados universitarios a partir de lo cual se demandaron recursos públicos y la creación universidades cofinanciadas por el Estado. Este hecho llevó, sin duda, a deslegitimar la presencia universitaria pública debido a su baja calidad, poco impacto investigativo, problemas internos, etc. Dicha situación permitió, entonces, que se legislara en pro de la creación de centros de educación superior de corte privado, una medida que formó parte del periodo de "modernización del Estado" establecido por el Consenso de Washington.

En consonancia con lo anterior, en este periodo se impusieron diversos proyectos legales que estuvieron alejadas del objetivo de fortalecer la universidad y que más bien obedecieron a oscuros intereses políticos. Pruebo de esto fue la depuración universitaria emprendida por el CES en esos años. La Unesco (1995) habla de la "pertinencia social de la universidad" y la "adecuación del ser y que hacer de la educación a su deber ser". Sin embargo, ser pertinente implica una institución efectivamente vinculada con su medio, que contribuya a producir las transformaciones necesarias para elevar el nivel de calidad de vida de su sociedad, de forma tal que sus conocimientos y producciones tengan efectivo impacto social. Así, pues, una universidad pertinente deberá desarrollar no solo proyectos de docencia e investigación, sino,además, incorporar proyectos de vinculación, y entre estos, entornos de aprendizaje enmarcados dentro de la praxis preprofesional.

\section{Tercera Reforma (2000 - 2010)}

Con el inicio del siglo XXI se presenciaron cambios acelerados en las estructuras productivas y la fuerte presencia de las tecnologías de la información y comunicación llevaron a los centros de enseñanza superior a adecuar sus procesos internos y sus ofertas académicas a un entorno virtual alineado con las necesidades más sociales frente a las cuales la universidad debe incidir. Ello se ve facilitado por el hecho de que la misma interconexión mundial hace que las universidades puedan conocer modelos de vinculación modernos en el marco de un aceleradointercambio de conocimientos.

Este entorno cambiante, y hasta cierto punto revolucionario, produce un nuevo orden jurídico que tiene como objetivo producir una sociedad del conocimiento. Para ello,en Ecuador se crean nuevas universidades destinadas a convertirse en referentes de cambio como Yachay, Ikiam y la Universidad de las artes.

Se trató en todo momento, dentro de esta reforma, de permitir a las universidades adaptarse a los cambios sociales y productivos en busca de una institución más identificada con la sociedad en vez de solo con los modelos empresariales, esto es, una "universidad solidaria y responsable" con su comunidad. Para conseguirlo, se pasó a concebir a la universidad como un todo en el cual participan empresarios, organizaciones sociales e instituciones culturales, bajo un marco de priorización de objetivos y metas, que articule empresa, universidad y comunidad. El problema de esta perspectiva es que losesfuerzos para generar la ansiada articulación han generado escasos proyectos de investigación universitaria, muchos de los cuales usaron fondos públicos, antes que privados, ya que las empresas no los vieron atractivos.

\section{Cuarta Reforma (2010 - 2020)}

En la actualidad está en curso una cuarta reforma universitaria que busca situar a la universidad en su entorno, mirando casa adentro sin perder de vista los cambios mundiales. Se trata de una especie de especialización del conocimiento a partir de sus insumos 
territoriales, con capacidad de adaptarse a las demandas de los ciudadanos.Para generar conocimiento que resulte pertinente es fundamental la sobrevivencia de las IES, así como respetar las realidades territoriales sin olvidar los cambios tecnológicos presentes. Todo esto es el objetivo a alcanzar. Para ello, la vinculación toma un rol protagónico al permitir que los sectores olvidados de la sociedad puedan acceder a beneficios derivados de la investigación, capacitación y formación. Se podría decir que lo que se exige a la universidad ecuatoriana en este contexto es que genere una nueva gestión del conocimiento basada en la integración de saberes y formas de aprendizaje formal, informal, presencial y virtual, realizada dentro de nuevos ambientes de aprendizaje. En función de esto, la acreditación de los indicadores viene a ser un importante punto de partida.

Uno de los aspectos centrales en el marco de la nueva dinámica de transformación académica que vive el Ecuador la constituye la participación activa de la universidad en los procesos de producción y transferencia del conocimiento al sector productivo y la comunidad. Este es el núcleo duro de los procesos de acreditación tanto de carreras profesionales como de las facultades, ahora denominadas"Unidades Académicas".

Son estos nuevos tiempos, cuya característica es la velocidad en las transformaciones científicas, los que exigen a las IES la renovación de sus prácticas investigativas de vinculación, que las tesis demuestren la pertinencia de los temas escogidos y que sus políticas de prácticas preprofesionales utilicenel conocimiento útil recibido en las aulas. Es así como el vínculo entre el mundo educativo y el productivo, no solo desde la visión empresarial, se vuelve un factor que fomenta cambios sustanciales en las mallas curriculares, en los programas de vinculación, en las redes científicas y en las aplicaciones prácticas de la teoría, todo ello en busca de una inserción laboral altamente calificada o de una capacidad de emprendimiento según altos niveles de exigencia. Es por ello que, tomando las palabras de López (2005, pág. 130), la vinculación no debe formar parte de una política particular, sino de una política global.

En este sentido, la visión global de la universidad debe apuntar a mejorar las estrategias de trabajo y de relación con la comunidad, algo que todas las univer- sidades del Ecuador vienen trabajando, so pena de no alcanzar la acreditación y desaparecer del sistema de educación superior. Bajo el argumento de la necesidad de vinculación se deja de lado el binomio instituciones educativas-empresas para trabajar el trinomio instituciones empresas-universidad-comunidad. Este es un proceso revolucionario que en algunas investigaciones se conoce como la triple hélice, aunque con la variante de que la comunidad es considerada como el Estado, según manifiesta Erkowitz\&Leydesdorff (2000). Para estos autores, el modelo pretende que el accionar de la universidad sea un creador del conocimiento, jugando un papel primordial entre la relación empresa y gobierno. Modelo que es un proceso intelectual orientado a visualizar la evolución de las relaciones entre universidad-sociedad. Es un modelo que ha recibido gran atención en el mundo occidental como un medio para fomentar la las innovaciones y el crecimiento, tareas pendientes de desarrollar en el sistema educativo superior del Ecuador.

Como se puede apreciar, es posible generar nuevas formas de concebir el papel de la universidad a partir de formas innovadoras de crecimiento económico y social, y el establecimiento de algunas estrategias metodológicas dirigidas a mejorar el vínculo de la universidad con su entorno social y productivo.

\section{ESTRATEGIAS METODOLÓGICAS PARA ESTABLECER VÍNCULOS EN EL CONTEXTO UNIVERSITARIO, LA EMPRESA Y LA COMUNIDAD}

El desafío actual para las universidades en Ecuador y en el mundo es forjar vínculos entre la universidad, la empresa y la comunidad. La vieja tendencia que consistía en una relación de afuera hacia adentro está cambiando por una planteada desde adentro hacia afuera. Ello busca afirmar una universidad más abierta y proactiva, que logre ser parte fundamental de los cambios en los territorios subnacionales.

Diversas son las formas para poder realizar un proceso de vinculación y son muchas las variables intervinientes. Revisando el análisis deRamírez (2013), quien habla del colonialismo universitario,es posible afianzar la perspectiva de este artículo a partir de la idea de que la generación de conocimiento y la verdadera riqueza de un país residen en la capacidad 
de sus universidades para articular la acción cooperativa del intelecto colectivo representado por el sector industrial, el Estado, los gobiernos locales, las cooperativas, los actores populares, los organismos no gubernamentales, los parques tecnológicos, etc. Esta tesis confirma lo expuesto a lo largo de este artículo en la medida que visualiza la separación del trabajo universitario de los sectores sociales comunitarios e incide en la actual necesidad de un acercamiento al sector empresarial.

En palabras de este autor la descolonización del sistema de educación superior se encuentra ligada a la ruptura con la creencia según la cual la universidad tiene el monopolio del conocimiento (Ramírez, 2013). En consecuencia es válido pensar que un paso importante consiste en que el conocimiento sea construido a partir de los saberes ancestrales y en base a todas las voces que pueda dar ideas y criterios sobre los problemas que afectan a las sociedades. En este proceso de planificación, el diagnóstico del contexto socio-laboral es fundamental, no solo según la inserción de los profesionales, sino también de su capacidad para emprender y ser gestores del cambio local. Así, se debe valorar que la posición de la universidad en su entorno ayudará a una mejor respuesta de esta a sus problemáticas.

A continuación, se definen algunos modelos de acción universitaria:

- Universidad tradicional: Este tipo de institución se rige bajo una lógica de puertas hacia adentro y plantea una oferta académica igual a la de otras con más años, aunque con poca capacidad de interrelacionarse, algo que con la nueva LOESes poco dable por causadel trabajo en red que se demanda.

- Universidad reactiva: Este tipo de institución lleva a cabo procesos ocasionales de vinculación ya sea conorganizaciones lucrativas o no. Estos hechos puntuales suelen responder a la presión territorial de parte de las autoridades locales antes que a planificaciones formales de intervención.

A partir de esta clasificación y según el estado de situación diagnóstica de la universidad, ella deberá tomar el mejor camino, amparada en el marco regulatorio que exista, en aras de contar con un modelo de gestión que incorpore de manera efectiva la vincula- ción universidad-empresa-comunidad en el contexto productivo y social propio de su ámbito de acción.

\section{MODELO DE GESTIÓN}

Definitivamente no existe un modelo de gestión que resulte perfecto para poder superar el déficit de la vinculación de la universidad-empresa-comunidad que arrastra la universidad ecuatoriana y latinoamericana en general. Si bien es cierto que existen importantes avances en países como Chile, Uruguay, Colombia, México y Argentina, haymucho trabajo que hacer en este tema. Ahora bien, pese a esto, es posible señalar algunas características que debería tener un modelo de gestión dirigida a formular una política de vinculación dentro deuna universidad. Tales lineamientos son mencionados a continuación:

- Una participación crítica y dinámica en conjunción con organizaciones comunitarias, empresariales, culturales y sociales, con observadores externos si fuera preciso y con flexibilidad para adaptarse a los cambios en el entorno productivo y social.

- Una gobernabilidad institucional mantenida a través de la conformación de un directorio en el que participen distintos representantes de las unidades académicas de la universidad y que posea amplias posibilidades de reprogramar y revisar sus planes, programas y proyectos de vinculación.

- Un financiamiento pararealizar actividades de vinculación, algo que también puede solventarse con ciertos recursos externos que permitan la viabilidad de lo planificado.

\section{CONCLUSIONES}

Para el mejoramiento de los procesos de vinculación es necesario profundizar el modelo, considerando algunos aspectos tales como:

1. Dimensión del entorno: El entorno productivo, social, comunitario y cultural demanda un trabajo considerable, que configura un espacio de acción fundamental para las instituciones educativas. Solo cabe mencionar que en las organizaciones empresariales la dependencia de la tecnología importada ocasiona que haya un divorcio con la universidad en cuanto a soluciones propias 
con elementos nacionales. En ese contexto, las universidades han dejado de lado su rol protagónico frente a otras universidades extranjeras. No obstante, esta debilidad puede servir para que estas encuentren una oportunidad de contribuir al desarrollo nacional, regional o local, con la creación de ciencia y tecnología no solo para la gran empresa, sino también para todo tipo de unidades productivas, sean estas populares o de economía solidaria, una orientación proveniente del $\mathrm{Su}$ makKawsay defendida por la Constitución del Ecuador.Obviamente, primero hay que superarla actual deslegitimación de la universidad como prestadora de servicios empresariales y tecnológicos y superar la desconfianza entre empresarios e investigadores.

2. Dimensión financiera: Las universidades se sustentan en estructuras de funcionamiento financiero casi inflexibles, por lo que la planificación de sus actividades de vinculación deben hacerse de manera participativa por lo complicado de los reajustes presupuestarios y la escasez de recursos. De ahí que en muchas ocasiones la capacidad de unir la universidad a proyectos de vinculación financiados desde afuera sea vital para aumentar su presencia territorial. Es, pues, vital contar con financiamiento para hacer una verdadera vinculación con impacto y con resultados visibles.

3. Dimensión institucional: Deben superarse las viejas limitaciones que tiene la universidad con respecto a los actores sociales y productivos, una limitación que nace de la escasa o nula comunicación con todos los actores del territorio (tal como se detalla en los resultados analizados en las encuestas). Esto ha ocasionado que la universidad no oriente adecuadamente sus proyectos y líneas de investigación a la resolución de problemas de alto impacto en la vida de los ciudadanos, así como de las empresas que demandan sus servicios. Por otra parte, también se debe agregar a lo anterior el desconocimiento de los procesos de trabajo en las instituciones y la escasa labor de los docentes, el cual expresa una falta de compromiso en el trabajo de equipo que exige la vinculación junto al respeto por las creencias, los saberes y los usosde actores no necesariamente poseedores de conocimientos técnicos propios del mundo académico.
En resumen, son estas las tres dimensiones que se deben considerar para poder hacer efectiva una verdadera vinculación de la universidad con la comunidad, acorde a los tiempos y con los lineamientos legales vigentes en Ecuador en estos últimos años.

\section{REFERENCIAS BIBLIOGRÁFICAS}

1. Ley Orgánica De Educación Superior. Art 107. (03 DE 2008). Obtenido de http://www.cortenacional.gob.ec/cnj/pmb/opac_css/index. php?lvl=categ_see\&id $=2935 \&$ main $=$

2. Asamblea Nacional del Ecuador. (12 de Octubre de 2010). Ley Orgánica de Educación Superior. Registro Oficial. Quito, Ecuador.

3. Consejo de Evaluación, Acreditación y Aseguramiento de la Calidad de la Educación CEAACES. (03 de 2015). Obtenido de http://www.ceaaces. gob.ec/sitio/wp-content/uploads/2013/10/ MODELO-GEN\%C3\%89RICO-DE-EVALUACI\%C3\%93N-DEL-ENTORNO-DE-APRENDIZAJE-CARRERAS-2-0-Marzo-2015-FINAL-pdf.pdf

4. ETZKOWITZ, H., \& LEYDESFORFF, L. (2000). From National Systems and "Mode 2" to a Triple Helix of University-Industry-Government Relations. The Dinamics of Innovation. pp. 109-123.

5. Ley Orgánica de Educación Superior, Registro Oficial 298. (12 de 10 de 2010). Obtenido de http://uide.edu.ec/SITE/norma_juridica.pdf

6. LOES. Art. 13. Literal a. (12 de 10 de 2010). Obtenido de http://uide.edu.ec/SITE/norma_juridica.pdf

7. LÓPEZ, S. (2005). La vinculación de la ciencia y la tecnoloía con el sector productivo. México: Universidad Autónoma de Sinaloa.

8. PEÑA, M. (2010). Obtenido de http://www2. ucsg.edu.ec/dmdocuments/Vinculacion-programas $\% 20$ de $\% 20$ vnculacion $\% 20$ de $\% 201 \mathrm{l} \% 20$ ucsg.pdf

9. PNBV. (2013). Plan Nacional del Buen Vivir. Quito: Semplades.

10.RAMÍREZ, R. (2013). Tercera ola de transformación de la educación superior en el Ecuador, hacia la constitucionalización de la sociedad del 
Buen Vivir.Quito: Dirección de Comunicación de la Secretaria Nacional de Educación Superior, Ciencia, Tegnología e Innovación (SENESCYT).
11.UNESCO (1995). Documento sobre políticas para el cambio y el desarrollo de la Educación Superior. París: Organización de las Naciones Unidas. 